\title{
Applying Information Visualization Techniques to Capture and Explore the Course of Cognitive Behavioral Therapy
}

\author{
Monika Lanzenberger and Silvia Miksch \\ Institute of Software Technology \\ and Interactive Systems \\ Vienna University of Technology \\ Favoritenstr. 9-11/188 \\ 1040 Vienna, Austria \\ monika@asgaard.tuwien.ac.at, \\ silvia@asgaard.tuwien.ac.at
}

\author{
Susanne Ohmann and Christian Popow \\ Department of Pediatrics \\ University of Vienna \\ Währinger Gürtel 18-20 \\ 1090 Vienna, Austria \\ susanne.ohmann@univie.ac.at, \\ popow@akh-wien.ac.at
}

\begin{abstract}
Tracking and especially comparing psychotherapeutic processes is a complex task involving a large number of individual and complexly related parameters. Therefore, descriptive and classical statistical methods are only suited for partial analyses. To overcome these limitations we introduce LinkVis, a new Information Visualization (InfoVis) tool used to visualize and evaluate psychotherapeutic processes. LinkVis is developed and clinically tested on the basis of Cognitive Behavioral Therapy treating anorectic girls. The user gets new insight into the data under investigation due to the combination of three different visualization techniques: scatterplots, Chernoff faces, and parallel coordinates. LinkVis supports exploring of complex timedependent data in order to gain more information about the psychotherapeutic process, especially when comparing different patients and groups.
\end{abstract}

\section{Keywords}

Exploration, information visualization, psychotherapeutic processes

\section{INTRODUCTION}

Psychotherapeutic processes are very challenging tasks and these processes need sophisticated methods to analyze the effectiveness, benefits, and limitations of various treatment options and to compare the treatment courses. Such analyses include a large amount of complex and time-dependent data, that are difficult to explore by descriptive and classical statistical methods. Therefore, in order to support the psychotherapists in observing, evaluating, and comparing the

Permission to make digital or hard copies of all or part of this work for personal or classroom use is granted without fee provided that copies are not made or distributed for profit or commercial advantage, and that copies bear this notice and the full citation on the first page. To copy otherwise, to republish, to post on servers or to redistribute to lists, requires prior specific permission and/or a fee.

SAC 2003, Melbourne, Florida, USA therapeutic progress, we introduce LinkVis, a new tool for visualizing complex psychotherapeutic plans and subplans in relation to the planned interventions, their execution and effects. The program aims at making the psychotherapeutic process accessible and comparable in and between individuals and groups at any given time point and according to its content and time-based dimensions. Mainly using evaluation data, our program is developed in parallel to a clinical study of anorectic girls but should also be applicable to other forms of psychotherapeutic research and quality control. In the following the main challenges are sketched:

- to gain new insights into the therapeutic process, in our project, by accompanying and comparing group and individual Cognitive Behavioral Therapy;

- to structure data and therapeutic plans in order to facilitate the standardization of treatment modules;

- to guide the supervision process;

- to simplify information transfer using temporal abstraction, task specific visualization methods, and task specific calculations;

- to support flexible calculation and visualization methods in order to meet the changing demands and needs of the users; and

- to create a bridge between text notes and formal representations.

In the next section, we illustrate the idea and approaches of InfoVis and show their benefits and limitations. In section 3, we explain the data used. The following two sections describe the users, their intentions, and the tasks of the program. Section 6 covers the visualization tool using a simple example to illustrate its functionality. Finally, we give an outline of the planned evaluation and formulate preliminary conclusions.

\section{RELATED WORK IN INFOVIS}

The goal of InfoVis is to promote a more intuitive and deeper level of understanding of the investigational data and to foster new insights into the underlying process $[18,19,20]$. InfoVis is the use of computer-supported, interactive, visual representations of abstract data in order to facilitate cogni- 
tion. It applies visual processing methods to abstract data and information and is a kind of external cognition - using resources in the world outside the mind in order to expand what the mind can do. An enormous amount of work has been done in the field of InfoVis in the last few years. The methods used range from geometric techniques (e.g., scatterplots, hyperslice [21], parallel coordinates [8]), icon-based techniques (e.g., Chernoff faces [2], stick figures [14]), pixeloriented techniques (e.g., recursive pattern techniques [10], spiral- and axes-techniques [9]) to interactive visualizations for hierarchical information (e.g., cone/cam tree [16]) and graph-based techniques (e.g., curved line) or hybrid techniques (arbitrary combinations of techniques mentioned). In addition, distortion techniques e.g., perspective wall [11], fisheye view [4] were explored. Most of these approaches [6, $7,13,15,22]$ focus on large amounts of multi-dimensional data. The challenges in designing visualization methods for time-oriented psychotherapeutic data is, however, quite different. The visualization tool should display the derived qualitative temporal patterns and important quantitative time-oriented values in a task-specific way and provide more information on a users' request. Data preprocessing is necessary for displaying the case- (or study-) relevant data as comprehensively and intuitively as possible [1, 5], eventually making necessary the reprocessing of the preprocessed data (e.g., data abstraction). This will result in a recursive and interactive concept [12].

\section{DATA CLASSIFICATION}

We distinguish between two kinds of data:

\section{static data}

It is requested only once and not changing over time (patient identification (ID), name, date of birth (DOB), therapy setting, patient history, begin of the disorder, diagnoses, type of anorexia, comorbidities).

\section{dynamic data}

Questionnaire items, body measures, estimated progress, etc. are collected throughout the study. These repeatedly collected data reflect the therapeutic course. There are 25 different questionnaires for each patient, 10 for the parents, and two for the therapists. As the various questionnaires are used repeatedly because the patients state changes over time (therapeutic process), this sums up to 150 questionnaires for every single patient. Typical questionnaires contain about 40 questions. There are also short questionnaires consisting of only three questions and a few large questionnaires with about 110 questions.

More exactly we deal with numerical data (integer values, usually 0-6), nominal data (clearly defined lists of attributes like 3 types of anorexia), dates (e.g., DOB), and free text. Numerical data are simple to handle and do not need to be converted for visualization. Nominal data and dates have to be converted into numerical values, and free text which usually is not suitable for visualization can be used as background information that is displayed on the users' request. Numerical data from a technical point of view are simple to handle but may be difficult to interpret. In such cases we have to analyze and define the way the therapist interprets the data. Although the meaning of numerical data is straight forward because ' 0 ' usually means 'Wrong' or 'No' and '6' means 'Right' or 'Yes', the interpretation of the whole data series may be difficult because the values cannot simply be compared if the questions and answers are different or inconsistent. Especially the representation of nominal data by numerical values can be misleading because the user tends to interpret numbers as ordinal numbers whereas a category represented by ' 1 ' does not have a direct relation to another category, e.g., '3'. Although the study contains many questionnaires, the amount of data per patient is quite small compared to other data sets like ECG or EMG monitoring data. Therefore, we use flat eXtendible Markup Language (XML) files for data storage and implementation. The universal format of XML is platform independent, easy and flexible to handle, compatible with standard formats of databases and sufficient for this kind of data. Every questionnaire is stored in a file and consists of pairs of data representing the question numbers and the associated values or text. The respective file names are created from the patient ID, the date and a questionnaire acronym.

Medical data have to be most carefully protected against misuse. Therefore, the whole system is password protected, the data transmission from the client to the server is SSL (Secure Socket Layer) encrypted, and identification data are separately stored with limited accessibility.

\section{USERS ...}

Visualization tools are strongly related to the users and their tasks. The tools offer specific possibilities and do not have general purposes. They support the exploration and investigation of specific data. We therefore need to know the intentions of the users.

LinkVis is used by two researchers (psychotherapists) exploring data based on a psychotherapeutic investigation. They are experts in medicine and psychology and familiar with computers. Their intention is to observe the therapeutic course and compare the results of group and individual therapy. Their most important goal is to help their patients, however in addition, they want to develop a structured method in order to analyze the questionnaires more thoroughly and to overcome the limitations of descriptive and classical statistical methods (compare Section 1). Therefore, they need to select and display interesting data sets of various patients or patient groups, in order to find new hypotheses (exploration) or to confirm or reject hypotheses and to gain more insight into the therapeutic process. The design of the user interface is therefore essential for supporting the data exploration and analyses tasks.

\section{5. ... AND THEIR TASKS}

As mentioned above, we see the tasks of the users as a basis for implementing tools and methods. Shneiderman developed the 'Visual Information Seeking Mantra' ('overview first, zoom and filter, then details on demand' multiple times) as a starting point for designing graphical user interfaces and for improving the interactivity of InfoVis techniques. Furthermore, he described a 'Task by Data Type 
Taxonomy' using seven data types and seven tasks [17]. Based on his approach, we identified four groups of tasks containing 14 specific actions, which we describe in the following.

\subsection{Choose, Combine, and Map Dimensions}

\section{Selecting dimensions for visualization}

Sometimes the user has specific questions of the questionnaires in mind, but mostly she will scroll through the lists of questions contained in the questionnaires. In order to build a specific data set she can choose up to 10 questions. A selected question is represented by one dimension of the visualization tool. The user may choose a keyword for labelling a specific question. Selecting dimensions is thus crucial for further examining the data.

\section{Combining and rating of questions}

The user can build-up a dimension by combining several questions, if she needs to consider more than 10 questions at a time. Several questions can be rated according to their effect on the aggregate value. The rating is based on a scale from '1' to '10' with whole-numbered graduations, where '10' means that the value is included unaltered, '9' means 90 percent included, etc. Only ordinal values are suitable for combining. However, combining and rating needs a thorough knowledge of the data.

\section{Rating of questions}

Besides the rating of combined questions a dimension could be rated as a whole. Some questionnaires address the patients and their parents, so it could be interesting to compare their answers. If the user wants to focus on the questions which were answered differently, she can give the others lower ratings. This results in attenuation of the variances of the unimportant values. These values can be confused with unaltered, low values. To prevent the user from misleading interpretations such values have to be marked, for example by color marking.

\section{Mapping questions to visual attributes}

After selecting a question, the user decides what visual attributes should represent these questions or dimensions. The user relates, for example, two questions with the axes of a scatter plot in order to display two dimensions at a time. She commits the axes of the parallel coordinates and the attributes of the Chernoff face. The question / dimension is labelled with a keyword for a later recall.

\section{Defining the scales}

The user defines the precision of the scale and the minimum and maximum values. According to this the data is displayed more or less in detail.

\subsection{Exploration}

\section{Visualizing the therapeutic course of a patient}

The user can gain important information by visualizing the therapeutic progress of a specific patient by comparing the state of the selected questions and looking for changes. Parallel coordinates and Chernoff faces seem to be convenient to display changes over time efficiently.

\section{Comparing family questionnaires}

The user selects questions addressing patients and parents in order to compare them. Patients with the diagnosis of anorexia nervosa sometimes have the tendency to give socially desired answers that do not match their real feelings. That does not implicate that their answer is wrong and the parents who answered the same questions differently are right. Therefore, such differences merit further investigation.

\section{Comparing the state of patients}

Typically the user wants to compare the state of the patients based on the data set she selected. Another question is to explore the influence of the group on the patients. This may be clarified by displaying data of the group members in parallel. If the user chooses Chernoff faces for visualizing patients states, the result cannot be predicted because Chernoff faces do not necessarily express happy or unhappy faces in relation to the patients state. In this case, parallel coordinates are possibly more convenient.

\subsection{Comparing and Classifying}

\section{Comparing two different visualizations}

If the data set is nearly identical, two different visualizations can be compared by highlighting the differences.

\section{Trends}

Striking changes during the therapeutic course are an important information for the user, unchanged data are less interesting. Then the selection of variables is supported by a list of the questions sorted by their variance. In addition, users can select less than 10 dimensions for visualizing trends.

\section{Details and overview}

Data can be displayed in various degrees of accuracy. The standard arrangement consists of 10 dimensions every one representing one question. The user can get an overview of the data, if the details are blinded out. She can do this by combining questions, or by displaying trends. If she wants to get more details she opens the context menu of a visualization attribute. This context menu offers access to every detail of the patient data.

\subsection{Handling Visualizations}

\section{Visualization history}

As details are often difficult to remember, the user can label specific visualizations by keywords for later recall.

\section{Storing data sets}

Some data sets are used very often. It is therefore useful to provide standard views and enable the user to easily adapt these standard views according to specific needs.

\section{Undo changes}

Every action of the user is stored for backup, enabling the user to change or repeat actions later on. 


\section{LINKVIS — LINKING INFOVIS TECH- NIQUES}

For the implementation of LinkVis, four issues are very important: (1) users, (2) tasks, (3) data, and (4) Basic Visualization Interactions. We need to focus on the users in order to understand their intentions and needs. We identified 14 tasks because it is the aim of the tool to support the user carrying out such tasks. We deal with specific data we described above. Finally, we apply the concept of 'Basic Visualization Interactions' as starting point [3] and use it as formal background. This is a framework for characterizing interactive visualization techniques. A Basic Visualization Interaction is described by its input, output, and operation. We distinguish three kinds of operations:

\section{Graphical operations}

can be divided into encode-data, set-graphical-value, and manipulate-objects. These operations change graphical attributes, change the mapping of graphical objects and data or operate on the graphical object as a unit of manipulation (e.g., zooming, coloring, ...) and affect the graphical representations of data objects (graphical state).

\section{Set operations}

expand the underlying data by classifying information and select data. These operations include creating, deleting, summarizing, joining, intersecting sets, and so forth. A set operation manipulates the data in order to create or alter a selection of data to be visualized. It affects the control state.

\section{Data operations}

affect the data state (database). Users can discover new facts during analysis or as mentioned above create useful classification information. It is useful to enrich the data with such new information contents.

Changing a state can cause secondary effects to other states. A set operation usually leads to a change of the graphical state. The manipulation of the graphical state could cause a modification of the control state and vice versa. An example is the handling of a graphical object in order to select data. Although there are some borderline cases which are difficult to relate to this structure, this framework is a good starting point for the implementation of LinkVis because it describes possible actions of the user. So we divided the user interface into three sections according to the three different types of operations defined above. The graphical operation section contains the visualization. In the set operation section the user can access buttons and sliders in order to select the data set. The database can be modified within the data operation section.

We can relate the above defined tasks with this concept because, although the levels of the tasks are different, every task contains such operations. Usually, there is one type of interaction that optimally characterizes a task. So far we did not define any tasks that modify the database itself. We therefore distinguish two groups of operations.

\section{Graphical operations:}

Map questions to visual attributes; define scales.

\section{Set operations:}

Select dimensions for visualization; combining and rating of questions; visualizing the therapeutic course of a patient; comparing family questionnaires; comparing the state of patients; visualization history; comparing two different visualizations; trends; details and overview; and storing data sets.

The task 'Undo changes' is not a visualization interaction and can therefore not be classified. It is not surprising that the most of the tasks are set operations, but if we focus on the manipulation of objects the border between graphical and set operations is not really strict.

LinkVis is based on three different techniques: Chernoff faces [2], scatter plots, and parallel coordinates [8]. These techniques are well known in InfoVis. Our tool offers the possibility to combine two of them. The user selects two methods for displaying the data set synchronously. Every technique offers advantages and disadvantages, moreover the data can be interpreted differently. If the user sees different aspects within the two visualizations, she will try to relate them and find analogies and contrasts. This offers an interesting potential for further exploration, especially to the advanced user. To give an impression of the benefits of our tool, we describe the techniques and their impact in detail followed by an example.

\section{Chernoff faces}

This face can represent up to 10 dimensions, e.g., by the size of eyes, slope of eyebrows, size and form of the mouth, and so forth. We tend to see the face as a whole, so this technique is very effective in communicating relative values and an overview. This kind of representation is based on the metaphor of emotions because the faces communicate different moods: Visualizing specific data sets creates happy, sorrowful, or angry faces. Interpretation of these faces may cause certain problems. If the user wants to visualize the state of a patient, the facial expression of the Chernoff face could mislead the user by identifying every happy face with a good state and every unhappy face with a bad state of the patient. The facial expression basically depends on the combination of questions selected by the user and the allocation to facial attributes. The data, even the data set of one patient could result in very different facial expressions. However, the advanced user is able to get important information at a glance comparing these faces easily and perceiving relative values.

\section{$2 D$ Scatter plots}

This technique is well known and widely used. Two dimensions / questions are represented by the values of the $\mathrm{x}$ - and $\mathrm{y}$-axis displayed as points or other symbols. In contrast to the Chernoff faces, absolute values are easily recognized by looking at the values on the axes, although the user will detect clusters or data holes firstly. Scatter plots communicate relative and absolute values effectively, but the number of dimensions is limited. Since we reserve size, shape, and 


\begin{tabular}{c|l|c|c|c|c|c|c}
\hline Dimension & Facial Attribute & Axis/Scatter plot & Axis Parallel coord. & Pat. A & Pat. B & Pat. C & Pat. D \\
\hline 1 & Pupils & x/1 & 1 & 1 & 1 & 2 & 1 \\
2 & Eyebrows & y/1 & 2 & 2 & 2 & 4 & 3 \\
3 & Mouth width & x/2 & 3 & 2 & 3 & 3 & 3 \\
4 & Eye size & y/2 & 4 & 3 & 2 & 3 & 4 \\
\hline
\end{tabular}

Table 1: Data set of 4 patients based on 4 questions / dimensions. Question 1: Do you often feel angry about your parents? Question 2: Do you feel strong and self-confident? Question 3: Do you like the way you look? Question 4: Do you talk to your colleagues about private problems?

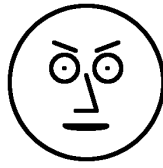

(a) Pat.A

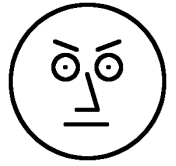

(b) Pat.B

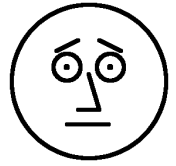

(c) Pat.C

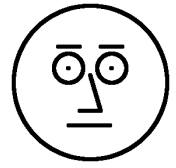

(d) Pat.D

Figure 1: Chernoff faces visualize the dimensions and values of Table 1, each describes a patient.

color of the symbol to provide the user with additional information, we need five scatter plots in order to display 10 dimensions by spatial arrangement. The user can configure grouping and size of the scatter plots, however, she tends to see five expressions separately. Users can not get an impression of the whole. Therefore, the combination of Chernoff faces and Scatter plots will provide additional information because every technique presents the data differently.

\section{Parallel coordinates}

In contrast to the $2 \mathrm{D}$ scatter plots, this technique places all axes vertically in parallel to each other. Thus we can display 10 dimensions on 10 axes. The data set is displayed by one line per record. We can rapidly record clusters of lines. Thus uncommon patterns will attract users attention. Arrangement of the axes is important. In order to analyze a record in detail, the line needs to be colored. Similar records will result in partly overlapping lines. It could be a problem that identical records are represented by a single line, and therefore, look like one record. Scatter plots cause the same problem. In contrast to Chernoff faces and scatter plots, parallel coordinates offer two important features: 10 dimensions can be combined in one graph. Similarly to the Chernoff faces, this method provides the user with overview information, but is not charged with the possibly confusing metaphor of emotions, especially if applied to psychotherapeutic data sets. In addition, the user can easily recognize absolute values.

Finally, we want to show an example based on four dimensions / questions in order to ease interpretation. It works the same way with 10 dimensions, but the user needs more experience to understand the representations. The unused attributes of the Chernoff face are shown, but do not represent any data. Table 1 shows a data set which represents four patients. The range of the values is '1'-'4' and means 'Never', 'Sometimes', 'Mostly', and 'Everytime'.

Every face of Figure 1 depicts the data set of a patient.

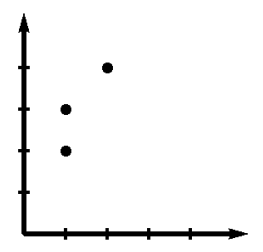

(a) x: Dimension 1 (Do you often feel angry about your parents?) / y: Dimension 2 (Do you feel strong and self-confident?)

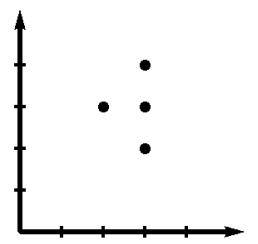

(b) x: Dimension 3 (Do you like the way you look?) / y: Dimension 4 (Do you talk to your colleagues about private problems?)

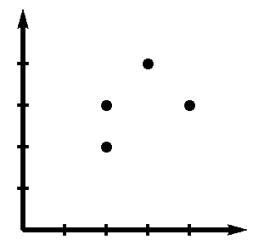

(c) $\mathrm{x}$ : Dimension 2 (Do you feel strong and self-confident?) / y: Dimension 4 (Do you talk to your colleagues about private problems?)

Figure 2: Scatter Plots visualize the dimensions and values of Table 1 .

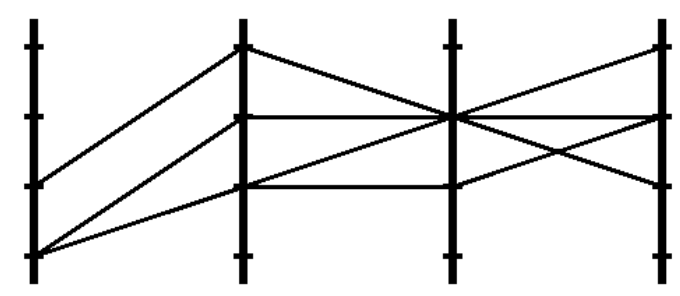

Figure 3: Parallel coordinates visualize the dimensions and values of Table 1 , axes 1 (left) to 4 (right): Do you often feel angry about your parents? Do you feel strong and self-confident? Do you like the way you look? Do you talk to your colleagues about private problems? 
The same data set is represented by scatter plots in Figure $2 \mathrm{a}$ and $2 \mathrm{~b}$ and by the parallel coordinates (compare Figure 3 ). The dimensions are modified in Figure $2 \mathrm{c}$ in order to show the combination of 'Question 2' and 'Question 4'. If the user selects Chernoff faces and parallel coordinates, she can compare the lines to the faces. As mentioned before she needs to highlight a line in order to view the details. The faces communicate an overview at the first glance, but the user could have problems to recognize that the eye size varies from 'Sometimes' to 'Everytime'. In contrast this information is shown clearly on the second axis of the parallel coordinates. On the other hand, the user sees easily the differences of the eyebrows in the Chernoff faces. The changes of the display caused by interactions of the user have an important impact to the exploration of data. The data set becomes more meaningful if the user examines the changes interactively. Our example demonstrates that flexibility and an interactive design of the user interface is very important for visualization tools.

\section{EVALUATION OF LINKVIS}

We plan to explore the functionality of our system on the basis of a clinical project visualizing the differences of group and individual Cognitive Behavioral Therapy of anorectic girls. The psychotherapists will select a couple of accepted and common hypotheses in order to prove the functionality of LinkVis. Thereafter, we plan to examine if LinkVis can also be used for other kinds of psychotherapy.

\section{CONCLUSION}

We developed a funded basis for the implementation of LinkVis which visualizes the course of psychotherapeutic data. The combination of different InfoVis techniques offers interesting potential for further explorations. Our project is an ongoing process. Detailed evaluation is needed, but first results indicate that LinkVis supports the exploration process of the psychotherapists effectively.

The strengths of LinkVis is its ability and functionality to interactively explore various dimensions of the complex timedependent data. LinkVis is especially oriented towards the characteristics of the psychotherapeutic data used - namely high-structured data instead of high-dimensional (complex structure, but few instances) data. Therefore, the interactive mapping and combining of data to visual components supports to get more and new insights into the data under investigation, which finally helps to overcome the limitation of descriptive and classical statistical methods. On the other hand, LinkVis is able to handle different tasks and users' demands and needs so that it guides the supervision process sufficiently.

\section{ACKNOWLEDGEMENTS}

The authors would like to thank the students Jutta Franz and Rene Almasy. Special thanks to Markus Rester for interesting comments and graphical and latex support.

\section{REFERENCES}

[1] S. Arndt, K. Lukoschek, and H. Schumann. Design of a visualization support tool for the representation of multi-dimensional data sets. In Proceedings of the
Fifth Eurographics Workshop on Visualization in Scientific Computing, (Rostock, Germany, May, 1994), pages 124-128, 1994.

[2] H. Chernoff. The use of faces to represent points in k-dimensional space graphically. Journal of the American Statistical Association, 68:361-368, 1973.

[3] M. Chuah and S. Roth. On the semantics of interactive visualization. In Proceedings IEEE Symposium on Information Visualization, (San Francisco, October 1996), pages 29-36, 1996.

[4] G. Furnas. Generalized fisheye views. In Proceedings of the ACM Conference on Human Factors in Computer Systems, (Boston, Massachusetts, U.S.A., April 1986), pages 16-23. ACM Press, 1986.

[5] N. Gershon. From Perception to Visualization, pages 129-139. Academic Press, London, UK, 1994.

[6] M. Gross, T. Sprenger, and J. Finger. Visualizing information on a sphere. In Proceedings of the 1997 Conference on Information Visualization, (October 1997), 1997.

[7] A. Inselberg. Multidimensional detective. In Proceedings of the 1997 Conference on Information Visualization, (October 1997), pages 100-107, 1997.

[8] A. Inselberg. The plane with parallel coordinates. Special Issue on Computational Geometry, 1:69-97, 1997.

[9] D. Keim. Pixel-oriented visualization techniques for exploring very large databases. Journal of Computational and Graphical Statistics, 5(1):58-77, 1996.

[10] D. Keim, H. Kriegel, and M. Ankerst. Recursive pattern: A technique for visualizing very large amounts of data. In Proceedings of the Visualization, (Atlanta, GA, 1985), pages 279-286. IEEE, 1985.

[11] J. Mackinlay, G. Robertson, and S. Card. The perspective wall: Detail and context smoothly integrated. In Proceedings of ACM CHI '91 Conference on Human Factors in Computing Systems, 1991, pages 173-179, 1991.

[12] S. Miksch and J. Gärtner. Fact-finding committee work: Data analysis beyond single sources. In G. E. Lasker and X. Liu, editors, Proceedings of the Proceedings of the International Symposium on Intelligent Data Analysis (IDA-95), (Baden-Baden, Germany, 1995), pages 124-128, 1995.

[13] S. Mukherjea, K. Hirata, and H. Yoshinori. Visualizing the results of multimedia web search engines. In Proceedings IEEE Symposium on Information Visualization 1996, pages 64-65, 1996.

[14] R. Pickett and G. Grinstein. Iconographic displays for visualizing multidimensional data. In Proceedings of the IEEE Conference on Systems, Man and Cybernetics, 1988, pages 514-519. IEEE Computer Society Press, 1988. 
[15] W. Purgathofer and H. Löffelmann. Selected new trends in scientific visualization. Technical Report TR-186-2-97-17, Vienna University of Technology, Computer Graphics, Visualisation and Animation Group, September 1997.

[16] G. Robertson, J. Mackinlay, and S. Card. Cone trees: Animated 3D visualizations of hierarchical information. In Proceedings of ACM CHI '91

Conference on Human Factors in Computing Systems, 1991, pages 189-194, 1991.

[17] B. Shneiderman. The eyes have it: A task by data type taxonomy for information visualizations. In Proceedings of the IEEE Symposium on Visual Languages, 1996, pages 336-343. IEEE Computer Society Press, 1996.

[18] E. Tufte. The Visual Display of Quantitative Information. CT: Graphics Press, Cheshire, 1983.

[19] E. Tufte. Envisioning Information. CT: Graphics Press, Cheshire, 1990.

[20] E. Tufte. Visual Explanation. CT: Graphics Press, Cheshire, 1997.

[21] J. van Wijk and R. van Liere. Hyperslice. In IEEE Proceedings of the Visualiation, (San Jose, CA 1993), pages 119-125, 1993.

[22] C. Ware. Information Visualization: Perception for Design. Morgan Kaufmann Publishers, 2000. 